\title{
Der Markt für Soziale Arbeit - Daten und Fakten
}

\section{DR. PIA JAEGER}

beschäftigt sich seit ihrer politikwissenschaftlichen Doktorarbeit mit dem Themenbereich Soziale Gerechtigkeit. Sie ist Referentin bei TUM University Press und lehrt an der Fakultät Sozialwesen der Dualen Hochschule in Stuttgart im Bereich Soziale Arbeit.

\author{
Ob in der Kinder- und Jugendhilfe, der \\ Schulsozialarbeit, in Gefängnissen, Krankenhäusern, \\ Gemeindeeinrichtungen, Flüchtlingsunterkünften, auf der \\ Straße und an vielen anderen Orten der Gesellschaft - \\ dass qualitativ hochwertige soziale Dienstleistungen \\ durch gut ausgebildetes Fachpersonal ausreichend \\ zur Verfügung stehen müssen ist ein elementarer \\ Bestandteil der gesellschaftlichen Infrastruktur.
}

In den letzten zehn Jahren haben Sozialarbeit, Sozialpädagogik und Sozialberatung auf dem Arbeitsmarkt rasant an Bedeutung gewonnen. 2017 waren rund 331.000 sozialversicherungspflichtig Beschäftigte in der Sozialen Arbeit tätig - ein Prozent mehr als im Jahr davor. Viele Sozialarbeiter*innen sind jedoch in Teilzeit tätig: Knapp jeder zweite Arbeitsplatz ist eine Teilzeitstelle. ${ }^{1}$

Etwa ein Drittel der Sozialarbeiter*innen arbeitet in einem klassischen Feld des Sozialwesens und jede(r) Fünfte in einer Heimeinrichtung, beispielsweise für Kinder, Jugendliche, Behinderte oder Senioren. Ungefähr ein Sechstel arbeitet im öffentlichen Dienst, also in Sozialämtern, Jugendämtern oder für einen Sozialversicherungsträger. Nur jede(r) zehnte Sozialpädagoge ist im Bildungswesen beschäftigt, beispielsweise als Schulsozialarbeiter*in in einer Ganztagsschule, oder bei einem Bildungsbetrieb, der eine Berufsvorbereitung und Berufsausbildung für benachteiligte Jugendliche oder Behinderte anbietet. Weitere beliebte Arbeitgeber sind Organisationen in Trägerschaft sozialer Interessenvertretungen, Kirchen und andere religiöse Vereinigungen und Krankenhäuser. ${ }^{2}$

Grundsätzlich gibt es für Sozialarbeiter*innen ein sehr breites Betätigungsfeld und es kommen immer wieder neue Bereiche hinzu. Durch die Unterzeichnung der UN-Behindertenkonvention beispielsweise, die das Ziel verfolgt, Menschen mit
Behinderung ein selbstbestimmtes Leben in der Gesellschaft zu ermöglichen, sind neue Tätigkeitsfelder entstanden. Managementaufgaben sind zu ihrem Aufgabenfeld hinzugekommen, oder auch die Tätigkeit als Fallmanager*innen für die Agentur für Arbeit. ${ }^{3}$

Auch wenn die Nachfrage nach Sozialarbeitern 2017 etwas nachgelassen hat, ist sie weiterhin auf einem hohen Niveau. Dies ist unter anderem auf die Betreuung von Flüchtlingen zurückzuführen. Insgesamt wurden 2017 um die 26.300 freie Arbeitsstellen bei der Bundesagentur für Arbeit gemeldet und damit 15 Prozent weniger als im Jahr davor. ${ }^{4}$

Im Sozialbereich werden viele Stellen im Rahmen von Projekten durch öffentliche Förderungen finanziert. Dies führt dazu, dass es einen hohen Anteil an befristeten Stellen gibt: etwa jede zweite Stelle ist befristet. Dies führt bei vielen Sozialarbeiter*innen zu Existenzängsten, auch wenn sie im Vergleich zu anderen akademischen Fachkräften relativ wenig von Arbeitslosigkeit betroffen waren.

Insgesamt waren im Jahr 2017 rund 6.300 Personen, die in der Sozialarbeit, der Sozialpädagogik oder -beratung eine Tätigkeit auf Fachhochschul- oder Hochschulniveau suchten, arbeitslos gemeldet. Dies waren zwar drei Prozent mehr als 2016, gleichzeitig aber der zweitniedrigste Bestand seit der Jahrtausendwende. Rechnerisch lag die Arbeitslosenquote nur bei 1,9 Prozent. 
Mittlerweile hat sich in der Sozialen Arbeit auch die Zahl der Erwerbstätigen mit einem akademischen Abschluss erhöht - seit 2007 um 36 Prozent. Hatten 2007 noch etwa 221.000 erwerbstätige Sozialarbeiter einen (Fach-)Hochschulabschluss so waren es 2016 bereits 300.000. Doch unabhängig vom formalen Bildungsabschluss sind im Bereich der Sozialen Arbeit viele Menschen mit hochqualifizierten Aufgaben betraut und das Stellenprofil entspricht einer akademischen Ausbildung. So waren 2016 rund 370.000 und davon 74 Prozent Frauen in den verschiedenen Bereichen der Sozialen Arbeit tätig und haben auch Leitungsaufgaben wahrgenommen. ${ }^{5}$

Dass Sozialberufe bei Schulabgängern sehr beliebt sind, zeigt sich an den $\mathrm{Ab}$ solventen- und Studienanfängerzahlen. 15.000 Studierende schlossen $2016 \mathrm{ihr}$ Studium ab. Insgesamt lag der Anteil der Bachelorabschlüsse im Sozialwesen bei 86 Prozent. Einer der Gründe sind die Beschäftigungsmöglichkeiten, die ein Bachelorabschluss Sozialarbeitern bietet. Die wenigsten Studierenden der Sozialen Arbeit sehen die Notwendigkeit für einen Masterabschluss, da es, abgesehen von einer Tätigkeit in der Forschung, kaum Anreize dafür gibt. Ein Masterabschluss wird in der Praxis weder zwingend gebraucht, noch sind damit größere finanzielle Vorteile verbunden.

Im Wintersemester 2016/17 waren rund 79.000 junge Menschen für ein Studium der Sozialen Arbeit, der Sozialpädagogik oder des allgemeinen Sozialwesens eingeschrieben - immerhin acht Prozent mehr als im Jahr davor. ${ }^{6}$

Sind die Sozialarbeiter jedoch erstmal in der Berufspraxis angekommen, erwartet sie ein geringes Einkommen, schwierige strukturelle Bedingungen, hohe Arbeitsbelastung und kaum adäquate gesellschaftliche Anerkennung. So verdienen etwa 64 Prozent der Sozialarbeiter*innen unter $2.000 €$ net$\mathrm{to}^{7}$, sind dafür aber beinahe täglich mit emotionalen Belastungen konfrontiert. In anderen Ländern ist die Situation ähnlich: Erwerbstätige in Fürsorgeberufen verdienen oftmals weniger als gleich qualifizierte Beschäftigte in anderen Berufen. ${ }^{8}$ Dies könnte unter anderem auch damit zusammenhängen, dass die Soziale Arbeit als ein sogenannter »Frauenberuf " gilt und damit, wie in anderen Bereichen auch, niedriger entlohnt wird. ${ }^{9}$
Tatsächlich sind etwa drei Viertel der Sozialarbeiter Frauen.

Auch Kathrin Dreffke, Fachstellenleiterin für minderjährige unbegleitete Flüchtlinge beim Jugendamt Gelsenkirchen, kann bestätigen, dass »der Beruf grundsätzlich sehr weiblich ist. Allerdings haben wir in unserem Haus gerade einen großen Zuwachs an männlichen Kollegen «, sagte die studierte Sozialpädagogin.

Auch sie sagt, dass die Arbeitsbedingungen in der Sozialen Arbeit nicht immer einfach sind. Es gebe überall zu wenig Personal, hohe Fluktuation und viele Fälle, die zu bearbeiten sind. Als Gründe sieht sie die gewandelten Verhältnisse in Deutschland: zum einem die Flüchtlinge, aber auch der Zuzug von Menschen aus Ost-Europa. Zudem seien die Menschen insgesamt aufmerksamer geworden - Erzieher, Lehrer und auch Nachbarn riefen viel schneller beim Jugendamt an. »Generell ist jedoch der Druck, die richtige Entscheidung im richtigen Moment zu treffen, sehr hoch."

Trotz schwieriger Bedingungen herrscht unter Sozialarbeiter*innen generell eine hohe Identifikation mit ihren beruflichen Aufgaben und ein starkes Verantwortungsgefühl gegenüber den Klienten. ${ }^{10}$

Dies erlebt Kathrin Dreffke ebenso. Ihre Arbeit mit den jungen Flüchtlingen ist bereichernd und sinnvoll und sie möchte »keinen anderen Job machen «. Sie habe das Glück, tolle Kollegen zu haben und viel Zusammenhalt zu erfahren. Dennoch müsse man sich sehr bewusst für einen Job in der Sozialen Arbeit entscheiden und sehr genau wissen, was man sich zumuten könne, sagt Dreffke.

Eine hoffentlich gute Nachricht ist, dass soziale Dienstleistungen endlich aufgewertet werden sollen. Die Streiks in den Jahren 2009 und 2015 im kommunalen Sozial- und Erziehungsdienst haben die Debatte zur Aufwertung der sogenannten SAHGE-Berufe (alle Berufe der Sozialen Arbeit, Haushaltsnahen Dienstleistungen und der Gesundheitsund Erziehungsbereiche) in den Fokus politischer und öffentlicher Diskussionen gerückt. Nicht zuletzt, weil den Streikenden eine Welle gesellschaftlicher Solidarität entgegenschlug. Hauptgrund ist jedoch der Fachkräftemangel. ${ }^{11}$ Mittlerweile steht die Aufwertung sozialer Dienstleistungen im Koalitionsvertrag der Bundesregierung.

\section{Anmerkungen}

(1) Statistik der Bundesagentur für Arbeit Berichte: Blickpunkt Arbeitsmarkt - Akademikerinnen und Akademiker, Nürnberg, Mai 2018, S. 98

(2) Statistik der Bundesagentur für Arbeit Berichte: Blickpunkt Arbeitsmarkt-Akademikerinnen und Akademiker, Nürnberg, Mai 2018, S. 98

(3) Orlanski, O.: Arbeitsmarkt der sozialen Berufe aus der Perspektive der Caritas, 2015, S. 16

(4) Statistik der Bundesagentur für Arbeit Berichte: Blickpunkt Arbeitsmarkt - Akademikerinnen und Akademiker, Nürnberg, Mai 2018, S. 100

(5) Statistik der Bundesagentur für Arbeit Berichte: Blickpunkt Arbeitsmarkt - Akademikerinnen und Akademiker, Nürnberg, Mai 2018, S. 98

(6) Statistik der Bundesagentur für Arbeit Berichte: Blickpunkt Arbeitsmarkt - Akademikerinnen und Akademiker, Nürnberg, Mai 2018, S. 101

(7) Henn, S., Lochner, B., MeinerTeubner, C.: Arbeitsbedingungen als Ausdruck gesellschaftlicher Anerkennung Sozialer Arbeit, Hrsg. Gewerkschaft Erziehung und Wissenschaft, Frankfurt am Main, April 2017, S. 29

(8) Hipp, L., Kelle, N.: Nur Luft und Liebe? Zur Entlohnung sozialer Dienstleistungsarbeit im Länderund Berufsvergleich, in ZSR 2016, 62(3), S. 238

(9) Henn, S., Lochner, B., MeinerTeubner, C.: Arbeitsbedingungen als Ausdruck gesellschaftlicher Anerkennung Sozialer Arbeit, Hrsg. Gewerkschaft Erziehung und Wissenschaft, Frankfurt am Main, April 2017, S. 29

(10) Henn, S., Lochner, B., MeinerTeubner, C.: Arbeitsbedingungen als Ausdruck gesellschaftlicher Anerkennung Sozialer Arbeit, Hrsg. Gewerkschaft Erziehung und Wissenschaft, Frankfurt am Main, April 2017, S. 51

(11) Schildmann, C., Voss, D.: Aufwertung von sozialen Dienstleistungen, in: Forschungsförderungsreport Nr. 4, Hrsg. Hans Böckler Stiftung, Düsseldorf, Juni 2018, S. 4 


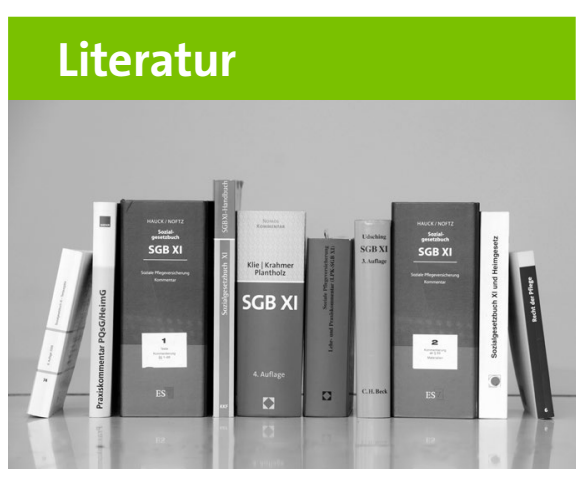

Bundesagentur für Arbeit (2018): Blickpunkt Arbeitsmarkt - Akademikerinnen und Akademiker, Nürnberg, Mai 2018

https://statistik.arbeitsagentur.de/StatischerContent/Arbeitsmarktberichte/Berufe/generische-Publikationen/Broschuere-Akademiker.pdf Henn, S., Lochner, B., Meiner-Teubner, C. (2017): Arbeitsbedingungen als Ausdruck gesellschaftlicher Anerkennung Sozialer Arbeit, Hrsg. Gewerkschaft Erziehung und Wissenschaft, Frankfurt am Main, April 2017

Hipp, L., Kelle, N. (2015): Nur Luft und Liebe? Zur Entlohnung sozialer Dienstleistungsarbeit im Länder- und Berufsvergleich, in: Zeitschrift für Sozialreform 2016, 62(3): 237-269

Orlanski, O. (2015): Arbeitsmarkt der sozialen Berufe aus der Perspektive der Caritas, in: die neue caritas spezial 2, Hrsg. Deutscher Caritasverband e.V., September 2015

Schildmann, C., Voss, D. (2018): Aufwertung von sozialen Dienstleistungen, in: Forschungsförderungsreport Nr. 4, Hrsg. Hans Böckler Stiftung, Düsseldorf, Juni 2018

\section{Pflegeberuf und Pflegeethik}

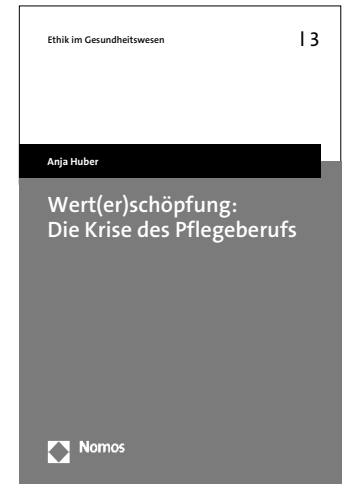

\section{Wert(er)schöpfung:} Die Krise des Pflegeberufs

Von Dr. Anja Huber

2019, 284 S., brosch., 54,- $€$

ISBN 978-3-8487-5844-9

(Ethik im Gesundheitswesen, Bd. 3) nomos-shop.de/41566

Pflegende ergreifen ihren Beruf, weil sie Menschen helfen wollen. Doch der ökonomische Druck im Gesundheitswesen hindert sie, Pflege entsprechend ihrem Berufsethos leisten zu können. Das führt zu Fluktuationen. Das Buch analysiert die Krise des Pflegeberufs und zeigt Wege aus der Krise auf.

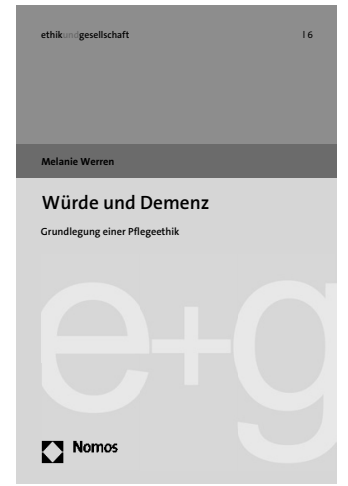

\section{Würde und Demenz \\ Grundlegung einer Pflegeethik Von Dr. Melanie Werren 2019, 244 S., brosch., 46,-€ ISBN 978-3-8487-5546-2 (ethikundgesellschaft, $B d .6$ ) nomos-shop.de/40601}

In diesem Buch wird eine pflegeethische Grundlegung der Würdekategorie geboten - exemplarisch dargestellt anhand der Würde von Menschen mit Demenz. Einerseits wird ein für ihre Situation angemessener Würdebegriff entwickelt. Andererseits werden daraus praktische Konsequenzen abgeleitet.

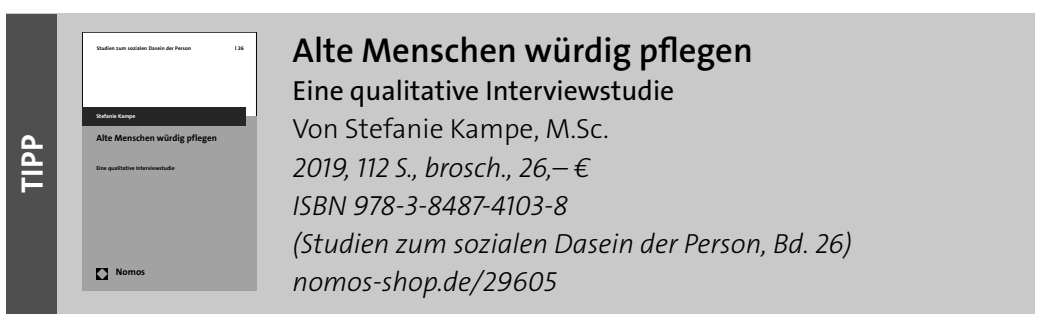

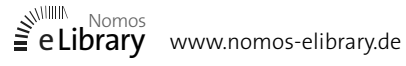

Bestellen Sie jetzt telefonisch unter (+49) 7221/2104-37. Portofreie Buch-Bestellungen unter www.nomos-shop.de Alle Preise inkl. Mehrwertsteuer 